\title{
PARTICIPACIÓN Y GESTIÓN DEL RIESGO DE DESASTRE: EXPERIENCIA DE PUERTAS NEGRAS EN VALPARAÍSO, CHILE
}

\author{
Felipe Mateluna ${ }^{1, *}$, María Constanza González ${ }^{2}$ y Raúl Díaz ${ }^{2}$
}

\section{RESUMEN}

Este trabajo aborda el desarrollo del Programa Quiero Mi Barrio en Puertas Negras, Valparaíso, Chile. En el 2017, los asentamientos regulares e irregulares de Puertas Negras sufrieron un devastador incendio que acabó con decenas de viviendas, y que demandó del Estado una estrategia diferente de reconstrucción que permitiera también recuperar el barrio de su abondo y vulnerabilidad ante desastres. El artículo entrega reflexiones sobre limitaciones y aciertos basadas en las experiencias de los profesionales del programa en el área, y de la comunidad. Ofrece luces sobre cómo la gestión del riesgo de desastre y la participación ciudadana, cuando son ejes centrales de las intervenciones, son positivamente reconocidos por los involucrados, pudiendo dar paso a un desarrollo más sostenible y seguro en asentamientos que han sido históricamente marginados. Se destaca la necesidad de mejorar funcionamiento institucional dentro del aparato estatal el cual tiende a crear retrasos en la ejecución de las intervenciones, provocando una brecha entre la confianza establecida por la comunidad y los tiempos de materialización. Así mismo, se ve positivamente el desarrollo de acuerdos entre el sector público y la academia para generar estudios y mecanismos novedosos que permitan involucrar a la comunidad dentro del programa y sus intervenciones.

\section{PALABRAS CLAVE}

Gestión del riesgo de desastre, Participación, Asentamientos informales, Programa Quiero Mi Barrio, Valparaíso, Chile

\section{PARTICIPATION AND DISASTER RISK MANAGEMENT: EXPERIENCE FROM PUERTAS NEGRAS IN VALPARAISO, CHILE}

\begin{abstract}
This paper addresses the disaster recovery and development of the neighborhood Puertas Negras in Valparaiso, Chile, through the Quiero Mi Barrio Program. In 2017, this settlement suffered a devastating wildfire that ended with dozens of housing units and demanded a public reconstruction strategy that would also enable the neighborhood to upgrade from its disaster vulnerability. The article provides constructive reflections on limitations and successes based on the experiences of program professionals and the community. It sheds lights on how disaster risk management and community participation, when they are at the core of interventions, are positively recognized by those involved, and can lead to a more sustainable and safer development in marginalized settlements. The need to improve state programming and public policies on urban development is also highlighted, which tend to affect trust of neighbors in the public system as interventions are often delayed. Finally, the development of agreements between the public sector and the academia in order to generate studies and innovative mechanisms for better community involvement in urban development programs and its interventions is positively seen.
\end{abstract}

\section{KEYWORDS}

Disaster risk management, Participation, Informal settlements, Quiero Mi Barrio Program, Valparaíso, Chile

\begin{abstract}
1. Secretaría Comunal de Planificación, Ilustre Municipalidad de Valparaíso, Valparaíso, Chile.

2. Programa Quiero Mi Barrio, Ilustre Municipalidad de Valparaiso, Secretaría Regional Ministerial de Vivienda y Urbanismo, Valparaíso. Chile.

*Autor de correspondencia: felipematelunafigueroa@ gmail.com
\end{abstract}

\section{RECIBIDO}

13 de mayo de 2019

\section{ACEPTADO}

2o de junio de 2019

\section{PUBLICADO \\ 1 de julio de 2019}

\section{Formato cita}

Recomendada (APA):

Mateluna, F., González M.C. \& Díaz, R. (2019). Participación y Gestión del Riesgo de Desastre: Experiencia de Puertas Negras en Valparaíso, Chile. Revista de Estudios Latinoamericanos sobre Reducción del Riesgo de Desastres REDER, 3(2), 97-108.

\section{@) $(\Phi \otimes$}

Todos los artículos publicados en REDER siguen una política de Acceso Abierto y se respaldan en una Licencia CreativeCommons Atribución-NoComercial 4.0 Internacional.

Revista de Estudios

Latinoamericanos sobre Reducción del Riesgo de Desastres (REDER)

Diseño: Lupe Bezzina Tipografía: Hospital 


\section{INTRODUCCIÓN}

Durante los últimos años Valparaíso, en Chile, ha sufrido una serie de desastres que han afectado de gran forma a la infraestructura urbana de la ciudad y la forma de habitar de la población que está asentada principalmente en los cerros de la comuna. Incendios, remociones en masa, movimientos sísmicos de gran magnitud, marejadas, entre otros, forman parte de los sucesos acontecidos en menos de una década en esta localidad.

La cantidad e intensidad de los desastres asociados a estos eventos extremos que ha experimentado la ciudad han puesto de manifiesto la necesidad de actuar para la reducción del riesgo con acciones mancomunadas entre los gobiernos nacionales y locales, las organizaciones sociales y los habitantes de territorio con el fin de generar estrategias que permitan minimizar el impacto urbano y social que actualmente han dejado estos eventos (Álvarez, 2007).

Junto a esto, el contexto social a nivel nacional ha posicionado a diversos movimientos sociales que han intentado poner en agenda de los gobiernos centrales un gran número de reivindicaciones ciudadanas. Chile ha mostrado una rearticulación de la población en torno a demandas pertinentes a mejorar la calidad de vida de los habitantes del territorio en materia de vivienda, urbanismo, previsión social, salud y educación principalmente.

Si bien para algunos la ciudadanía actual puede interpretarse como individualista, esta también debe ser entendida cada vez más como conjunto de individuos, debido a su capacidad de organización y participación ciudadana, manifestada especialmente en Chile en las últimas décadas a través de la demanda constante por participar en diversos procesos administrativos de la planificación de las ciudades y las políticas públicas.

La ciudadanía no debe ser considerada simplemente como una categoría formal que enumera los titulares de derechos, ni tampoco como un sujeto real o potencial, hoy "la ciudadanía incluye a quienes poseen o bien reclaman derechos y participan de la comunicación política" (Cheresky, 2008, p.17).

El concepto de 'ciudadanía' ha tomado gran relevancia en la comuna de Valparaíso con la irrupción de una administración política en la zona que ha posicionado este término como el pilar de su forma de ejecutar el gobierno local. Hoy en la ciudad, se expresa que "esta nueva ciudadanía no agota su existencia en el pronunciamiento electoral. Las elecciones y la legalidad de la representación que emana de ese pronunciamiento se han enraizado y son el sustento de la existencia de democracias efectivas pese a todas las contingencias institucionales y sociales" (Cheresky, 2008, p.19).

La importancia de la ciudadanía no radica tan sólo en su pronunciamiento en las elecciones, también se expresa porque desde ella hoy son capaces de nacer nuevas alternativas de hacer política social y pensar la planificación urbana de los territorios. Las participaciones que los habitantes de la comuna están desarrollando en el marco de, Plan Comunal de Desarrollo (PLADECO), Plan Almendral, Programa Quiero Mi Barrio Puertas Negras, Parque el Litre, entre otros, permitirá generar planes de inversión y de gestión del riesgo de desastre desde una base ciudadana.

Además, como lo plantea García (2014), los habitantes del territorio están mostrado un "mayor interés por aspectos cercanos y puntuales de la ciudad, programas de participación de gobiernos locales con organizaciones de base y ONG's, asociaciones intermunicipales, para generar 'corredores productivos' o entes intermunicipales, planificación estratégica en ciudades grandes e intermedias, presupuestos participativos, etc." (García, 2014, p.10).

Tras el 'Mega-incendio' de Valparaíso ocurrido el 2014, con miles de afectados en más de 5 cerros de la ciudad (unas 800 hectáreas) y 15 fallecidos, se levantaron diversos procesos de reconstrucción en los sectores afectados, suscitándose durante la planificación diversas problemáticas debido a los lugares de asentamiento de la población y las condiciones que dificultan un ordenamiento territorial. Más recientemente, durante el 2017, un nuevo gran incendio afectó esta vez el sector consolidado de Puertas Negra, y los asentamientos informales o irregulares en Los Lancheros, quemando un centenar de casas y dejando a cientos de personas damnificadas en la periferia de la ciudad, como se aprecia en la Figura 1.

A la fecha, el gobierno ha intentado dar respuesta a la serie de demandas en materia de vivienda e infraestructura urbana que afectan a la zona. Es en este contexto que el Programa Quiero Mi Barrio (PQMB) - perteneciente al Ministerio de Vivienda y Urbanismo (MINVU) y 
ejecutado a través del municipio local- ingresó a Puertas Negras hacia fines del 2017 con el objetivo de generar espacios públicos que aportaran a mejorar el entramado urbano del lugar.

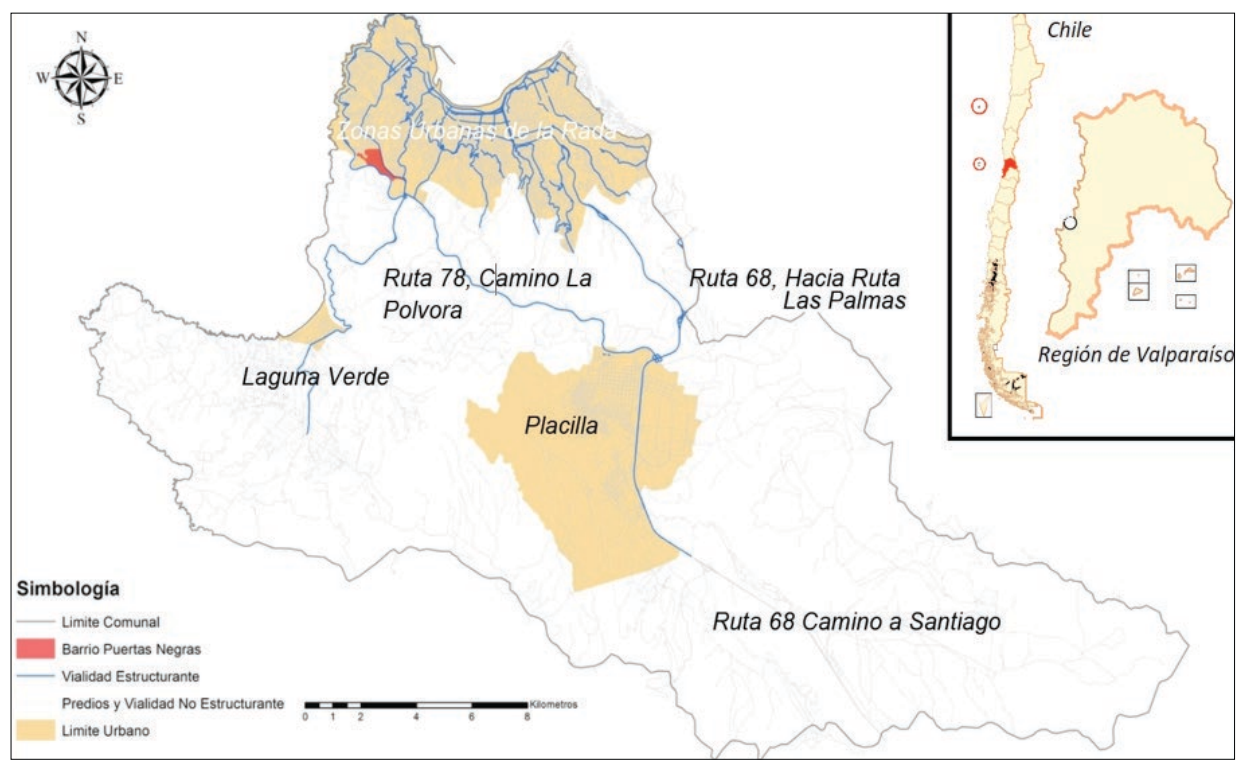

Figura 1. Mapa Zonas Urbanas de Valparaíso y Rutas Estructurantes Intercomunales Fuente: Departamento de SIG, I. Municipalidad de Valparaíso, 2018.

Este Programa de Recuperación de Barrios del MINVU, nació el 2006 con el fin de mejorar la calidad de vida de las personas a través de un proceso participativo que involucra al municipio y la propia comunidad beneficiada, permitiendo así la recuperación de los espacios públicos, el equipamiento y el fortalecimiento del tejido social (PQMB, 2019).

En la primera fase de implementación del programa en Puertas Negras, se desarrolló un plan maestro y se priorizaron las obras relacionadas a mejorar los accesos del sector, entendiendo por parte de la comunidad la importancia de generar mejores vías de evacuación ante una catástrofe, junto a propiciar el ingreso de vehículos de emergencia al sector a través de calles que cumplan con las condiciones normativas mínimas.

Mediante la ejecución municipal del PQMB Puertas Negras se establecieron vínculos con la Universidad de Valparaíso, a través de su Centro de Investigación de Vulnerabilidades e Informalidades Territoriales (CINVIT), para trabajar la gestión del riesgo de desastre (GRD) de forma participativa con la comunidad, permitiendo así una vinculación directa entre la ciudadanía y esta temática. Desde entonces, la GRD se ha posicionado como el eje de la ejecución de este programa de inversión en su segunda fase. Bajo la alianza entre la academia, los equipos municipales y ministeriales, se ha desarrollado un trabajo comunitario en torno a visibilizar los principales factores de riesgo en el sector, levantados gracias a la información entregada por los vecinos y vecinas en una primera instancia.

Velar por la reducción del riesgo de desastre (RRD) debe ser una prioridad nacional y local, tal como fue establecido en el Marco de Acción de Hyogo 2005-2015 y reafirmado recientemente con el Marco de Sendai 2015-2030 (UNISDR, 2015), para ello la participación de las comunidades es de suma relevancia a la hora de establecer políticas especificas y gestionar recursos para abordar esta materia. La participación ciudadana se define como "la relación que se establece entre los individuos en su calidad de ciudadanos y el gobierno municipal o local con el fin de hacer valer sus derechos, responder a sus deberes e influir favorablemente en sus políticas y funcionamiento". (Álvarez, 2007)

En el sector de Puertas Negras en Valparaíso, la ocurrencia de desastres socionaturales no es un suceso aislado, al igual que en el resto de la comuna. Los asentamientos presentan construcciones en laderas de material ligero y con escasas obras de habilitación, sumado a la existencia de microbasurales y condiciones climáticas adversas durante épocas de verano e invierno, que propician además la generación de incendios durante los meses de verano (diciembre, enero y febrero), y remociones en masa o inundaciones en invierno (junio, julio y agosto). 
Los esfuerzos en la RRD deben ser sistemáticos en las distintas escalas de la aplicación de políticas públicas, desde el desarrollo de programas como el PQMB, hasta la planificación general de las políticas sociales y urbanísticas del país. Es bajo este enfoque que la temática se ha posicionado como el articulador de la planificación que desarrolla el equipo municipal en el territorio de Puertas Negras, buscando potenciar la participación de vecinos en las distintas tareas del programa.

En resumen, este trabajo aborda el desarrollo del PQMB en Puertas Negras, el cual sufrió un devastador incendio que acabó con decenas de viviendas, y que demandó por parte del Estado una estrategia diferente de reconstrucción que permitiera también recuperar el barrio de su abondo y vulnerabilidad ante desastres. En las siguientes secciones se abordan las diferentes limitaciones y aciertos durante el desarrollo del programa, y se ofrecen reflexiones que pueden servir para mirar de forma constructiva la integración de la GRD, RRD y participación ciudadana como eje central de las intervenciones de desarrollo urbano.

\section{GESTIÓN DEL RIESGO DE DESASTRE: COMUNIDAD Y ACCIONAR INSTITUCIONAL}

A partir del incendio 2017 el municipio de Valparaíso desarrolló un trabajo multidisciplinario en torno a la elaboración del primer plan maestro de prevención de incendios cuyo objetivo implicó realizar una planificación estratégica basada en la visión común y participativa de la comunidad, con el fin de aplicar iniciativas al corto, mediano y largo plazo, dentro del ámbito institucional, social, ambiental y urbano. En resumen, se buscó reducir vulnerabilidades y el riego de incendios dentro de la ciudad, con la aplicación de iniciativas en los diferentes niveles del ciclo de riesgos de desastre.

El plan maestro integró la mirada de la ciudadanía, logrando la identificación y priorización de áreas de riesgo en base a tres ejes: el ordenamiento territorial, la intervención comunitaria, y la prevención para la emergencia (ver Figura 2). Con ello, se desarrollaron mapas comunales de zonificación de interfaz urbano-forestal, priorización por vulnerabilidad social de unidades vecinales y tiempo de respuesta hacia áreas de mayor riesgo, entre otros (Municipalidad de Valparaíso, 2018).

Los resultados del estudio previo al plan maestro permitieron constatar que el sector de Puertas Negras presenta alta vulnerabilidad en los tres ejes investigados (ordenamiento, comunitaria, y prevención), posicionándolo como un punto importante para trabajar de manera coordinada entre las instituciones y las comunidades con el fin de disminuir los riesgos que presenta la comuna.
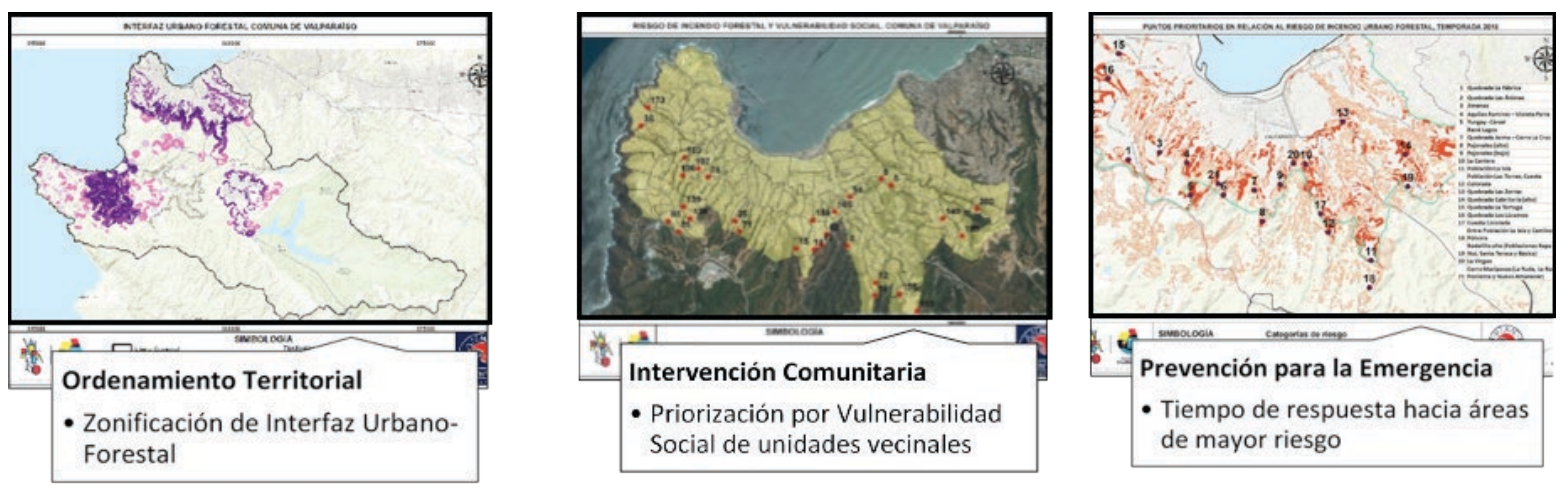

Figura 2. Tres ejes investigados en el Plan Maestro para la Gestión del Riesgo de Incendios Fuente: Municipalidad de Valparaíso, 2018.

Las zonas de interfaz urbano-rural forestal representan una especial preocupación debido a que son las áreas de mayor riesgo de incendio en la comuna. Dentro de las zonas de interfaz definidas, dos resaltan: 'Intermix', es decir, cuando las viviendas están insertan dentro de la vegetación forestal; y la de 'Interfaz no consolidada', en la cual las viviendas se encuentran en contacto con extensiones de vegetación.

La quebrada La Fábrica toma especial protagonismo como límite sur poniente del barrio e hito geográfico que influye en el territorio. Esta presenta problemáticas respecto a su estado de conservación dado que se encuentra alterado a causa de la presencia de viviendas en sus laderas, la modificación de su estrato vegetal producto del incendio y la existencia de micro-basurales a lo largo de su extensión (PQMB, 2018a).

Luego del incendio ha disminuido la vegetación que caracterizaba a la quebrada La Fábrica (ver Figura 3), alterando su apariencia, dado que su cobertura vegetal se estimaba en un $75 \%$ 
antes de la catástrofe, llegando a casi a un $0 \%$ post-incendio, lo cual refleja la magnitud del daño (Bellec \& Draghi, 2017). Es relevante señalar, que la quebrada La Fábrica posee una alta densidad de viviendas en conjunto con la presencia de vegetación, lo cual indica un riesgo para la población dado que no se realiza un manejo de la carga combustible que se produce entre los medios urbano-forestales.

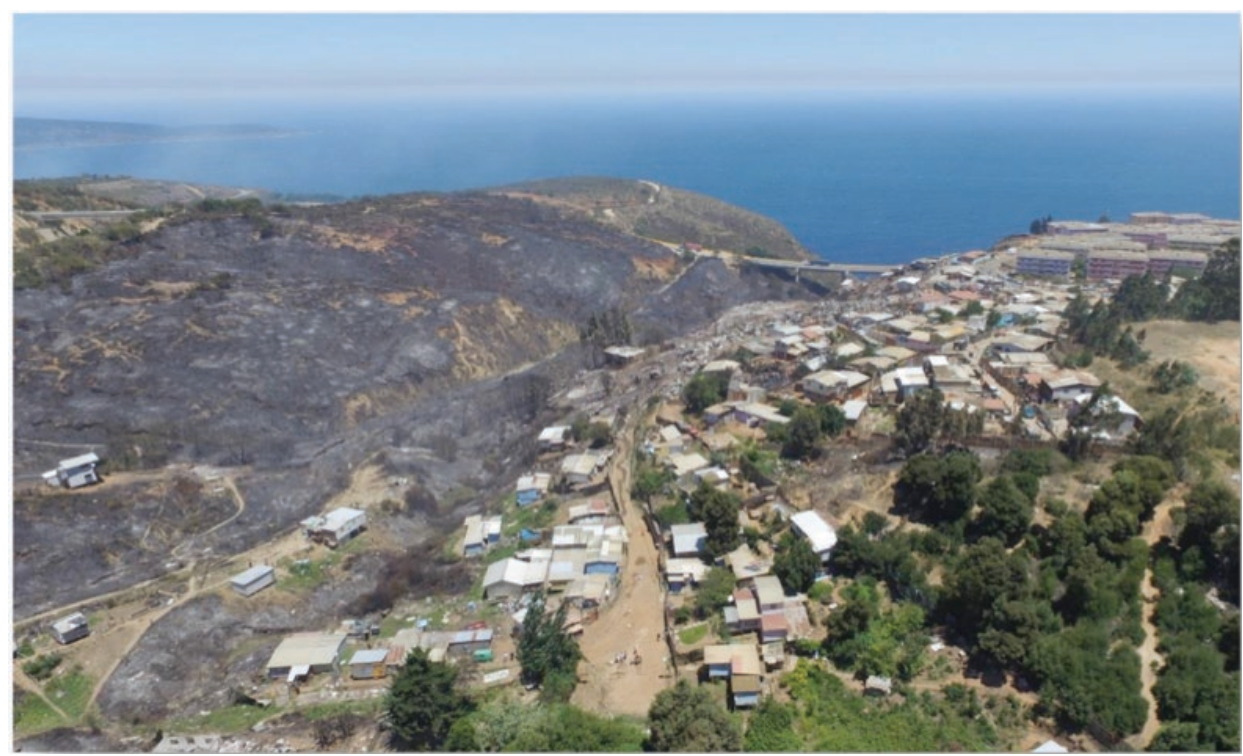

Figura 3. Fotografía Aérea Quebrada la Fabrica tras Incendio de Enero del 2017

Fuente: Serviu, Región de Valparaíso, 2017.

Las conectividades del barrio, su articulación con las vías estructurantes de la ciudad y su ubicación en respecto a zonas de la comuna de alto desarrollo urbano, forestal e industrial, se encuentra en relación directa con las zonas de alta producción forestal del sector de Laguna Verde, como con la zona urbano industrial en expansión del sector de Placilla (ver Figura 1).

Estos dos sectores y sus interfaces impactan directamente al barrio a través del desarrollo vial industrial que permite el intercambio económico del país. En este sentido, el Acceso Sur se consolida como uno de los límites más relevantes del polígono, perfilando el medio natural de la quebrada La Fábrica, a la vez que constituye un quiebre en la relación del barrio con el mar que históricamente se daba a través de Los Acantilados.

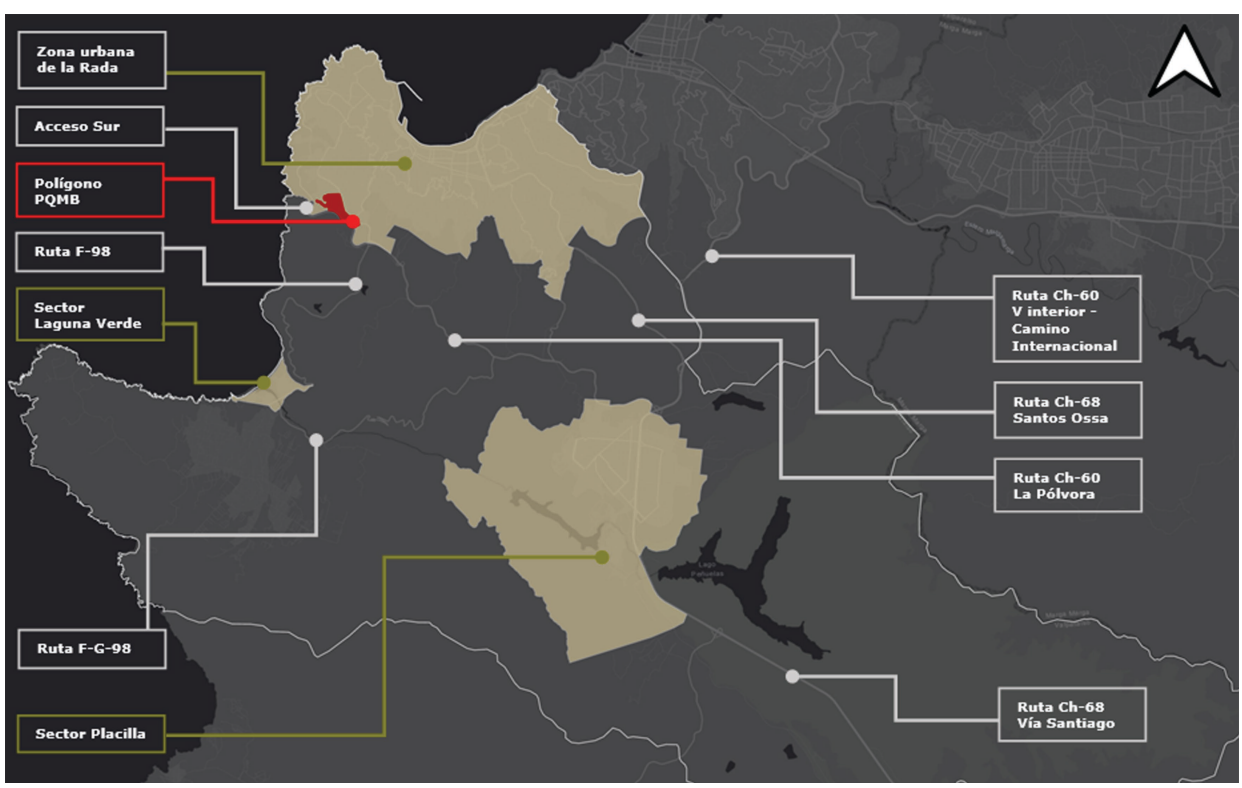

Figura 4. Mapa comunal y principales conectividades Fuente: Autores, 2019. 
Según los relatos de los habitantes del sector recogidos en los talleres y marchas exploratorias realizadas por el Programa Quiero mi Barrio (PQMB), la vinculación con el espacio natural de la quebrada constituía la única forma de esparcimiento recreativo y, al mismo tiempo, una fuente de trabajo especialmente vinculada a la zona poniente del polígono, lo que actualmente se conoce como Campamento Los Lancheros del Bongo, cuyos habitantes desarrollaban labores relacionadas con el mar y utilizaban el espacio como un camino natural hacia el océano Pacífico. En la Figura 4 podemos aprecias las conectividades principales a nivel comunal que se relacionan con el polígono de ejecución del programa.

La vinculación entre medio construido y medio natural forestal ha dejado expuesto al territorio a diversos incendios forestales, de los cuales el mayor aconteció el 2 de enero de 2017, declarándose 'Zona de Catástrofe' a una parte importante del polígono, considerando viviendas emplazas en asentamientos informales y consolidadas, afectando a más de un centenar de viviendas como se grafica en la Figura 5. Lo anterior dejó en evidencia la vulnerabilidad del territorio frente a desastres socionaturales y ha delatado cómo la falta de mitigación acentúa el riesgo de ocurrencia de nuevos eventos similares.

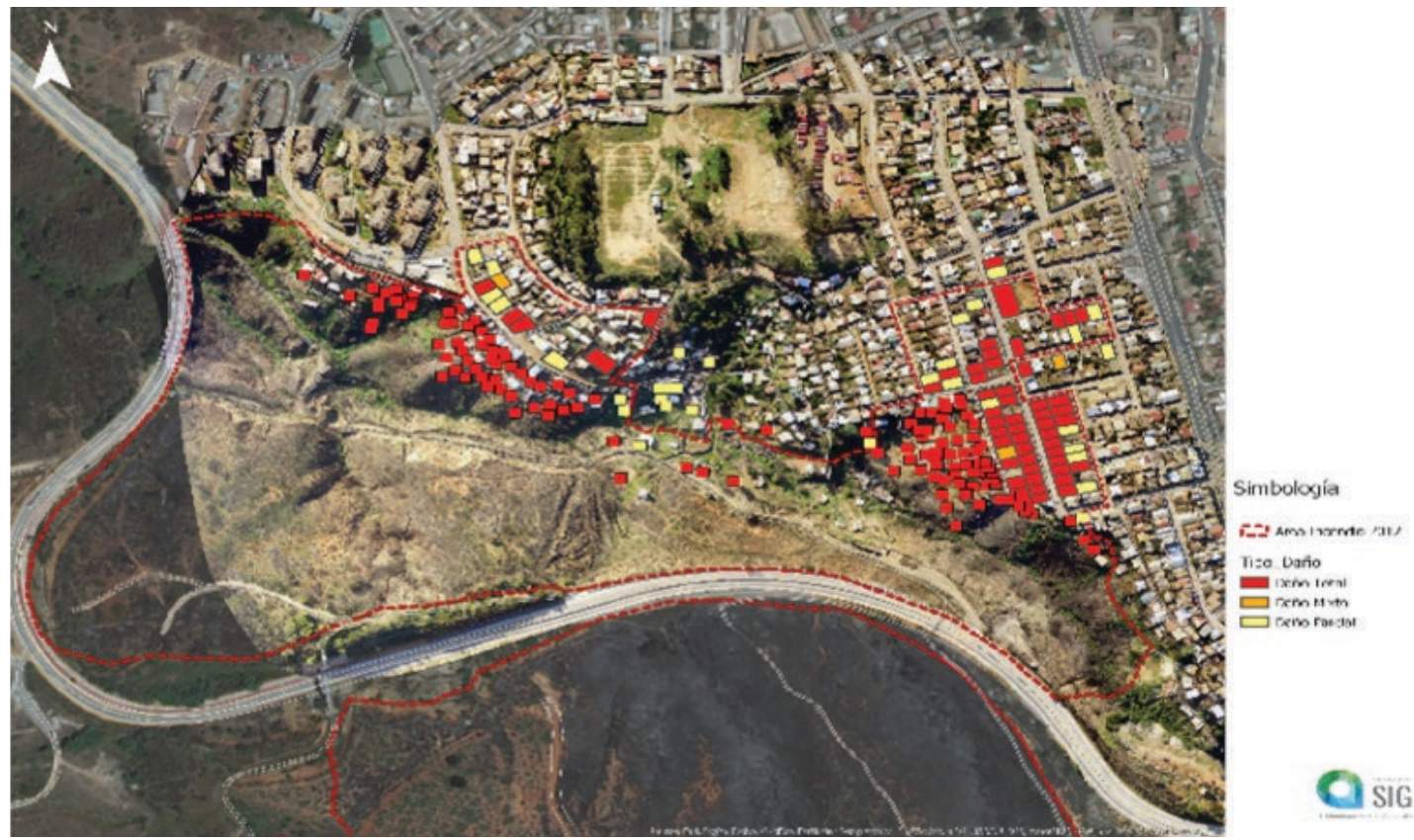

Figura 5. Mapa Satelital viviendas afectadas por incendio de Puertas Negras, Valparaíso, Chile Fuente: Fuente: Informe Final, Fase I, PQMB, 2018c.

El desarrollo urbano e industrial hacia el sector de Placilla de Peñuelas ha contribuido a la existencia de un gran flujo vial, desde y hacia este sector a través de Avenida Baden Powell, adyacente al barrio Puertas Negras y principal vía conectora del barrio (PQMB, 2018c). Si bien se realizaron obras de mejoramiento vial en esta avenida luego del incendio, éstas no contemplaron vincularse con los ejes viales interiores del barrio llegando incluso a cerrarse algunos de los accesos de la zona denominada Los Fleteros, acentuando la desagregación urbana del sector y generando un importante riesgo para la evacuación ante un nuevo evento natural.

Por su parte, en la vía por donde se accede a Valparaíso desde el Camino La Pólvora, hacia la ladera poniente de Pueblo Hundido, se ha conformado un micro-basural importante, con la consecuente degradación del medio natural y el riesgo de incendio de la quebrada La Fábrica y el sector consolidado de la Población Joaquín Edward Bello.

La vinculación del barrio con su entorno inmediato, es decir, poblaciones aledañas y sus respectivos equipamientos, se ve tensionada por el estado de las vías internas del sector, dado que desde algunos puntos del polígono se dificulta el desplazamiento hacia otros lugares de la parte alta del cerro Playa Ancha. Esta situación tiende a afectar la cotidianidad, convivencia, utilización de espacios públicos y de equipamientos que se identifican cercanos al barrio.

De acuerdo a la información recabada en instancias de diagnóstico como los recorridos barriales, fue posible identificar las problemáticas generadas por la fragmentación del territorio: la 
conformación de espacios islas dentro del polígono, los cuales se conectan de forma difusa con la trama regular del barrio; recorridos peatonales de baja calidad urbana, que han sido desarrollados por los propios habitantes de forma progresiva y espontánea, con fin de generar movilidades desde y hacia las zonas de conectividad tanto intra-barriales como comunales (PQMB, 2018a). Según lo observado, estas movilidades -con una trama urbana desagregada- dificultan y afectan directamente el uso cotidiano de los distintos espacios públicos que se encuentran dentro o cercanos al barrio, como jardines infantiles, escuelas, servicios de salud, equipamiento de comercio entre otros.

En este sentido se toma en consideración la investigación desarrollada en el marco del Proyecto FONDECYT N¹090198, titulado "Movilidad Cotidiana Urbana y Exclusión Social Urbana", que da cuenta de cómo se vive y se hace el espacio social en su cotidianeidad, entendiendo que existen experiencias, significaciones, valores, que van dando sentido de identidad en los sujetos que la componen, y explican las relaciones y prácticas sociales que se dan en el espacio. Esta muestra que la configuración del espacio social es vulnerable en dos sentidos: primero, por la distribución del espacio geográfico-territorial $y$, en segundo, por el 'acceso' a la ciudad, vale decir existen condiciones materiales y subjetivas que generan segregación social en cuanto a la construcción de la ciudad (Jirón, 2009)

La comprensión de estos conceptos nos permite graficar las complejidades de un territorio que se funda a partir de otra catástrofe,

"Gestada a partir de una vía institucional, la población Puertas Negras nace como parte de la Operación Emergencia implementada en Valparaíso luego del terremoto de 1965. De esta forma, el movimiento telúrico, que afectó a la provincia el 28 de marzo de ese año, fue el elemento circunstancial que generó el terreno propicio donde mezclar la aplicación de estrategias habitacionales oficiales, y los intereses individuales de cada componente de este futuro cuerpo colectivo, con miras a encontrar una pronta solución al problema".

Urbina $(1997$, p.4)

La respuesta institucional nace de forma inmediata ante la tragedia, sin embargo, son los propios pobladores los que impulsan a través del trabajo colectivo las mejoras urbanas mínimas para la instalación de sus viviendas, "En adelante, estas incipientes agrupaciones vecinales desarrollaron acciones que pretendían fabricar su entorno, mejorando su condición urbana por medio de la división básica del trabajo familiar, y la colectivización de la labor urbana" (Urbina, 1997, p.6).

Bajo esta forma de trabajo se lleva a cabo un proceso de 'colonización' de un territorio inhóspito que fue conformándose proporcionalmente en la medida del avance de la organización colectiva. "Se establecen las primeras aproximaciones entre el grupo vecinal y la 'élite dirigencial' en pos de trabajos de alcantarillado, alumbrado público u otro tipo de experiencia que requiera del trabajo colectivo organizado" (Urbina, 1997, p.6).

Finalmente, el Estado asume un rol distante en la participación del desarrollo urbano del sector y sólo a partir del progreso generado por la comunidad organizada se logra situar a los pobladores en una suerte de equilibrio ante la institucionalidad, de quien demandan las soluciones para mejorar las condiciones del territorio (Urbina, 1997). Ante esta ausencia histórica institucional, con la escasa planificación frente a una catástrofe y la evolución de la organización comunitaria en el territorio, hoy se puede determinar la sensibilidad de un sector altamente vulnerable en su contexto urbano-habitacional, con viviendas de emergencias (llamadas 'mediaguas' en Chile) que se convirtieron en viviendas definitivas sin cumplir con la normativa vigente de construcción chilena.

Hoy en día, tras la última catástrofe que afecto al territorio el 2017, la institucionalidad ha tenido un rol más activo, con iniciativas como el proceso de reconstrucción en el sector más consolidado de la población, a partir de la aplicación de subsidios post-catástrofe con financiamiento diferenciado a los subsidios regulares, línea de intervención desarrollada por al Servicios de Vivienda y Construcción (SERVIU).

Por otro lado, el municipio en conjunto con la Programa Campamento ha desarrollado una línea de intervención en los asentamientos informales que fueron afectados por el incendio con el financiamiento de estudios de uso y prospección de suelo en Puertas Negras. Esto permitirá el desarrollo de un proyecto de recuperación de espacios que impedirá la reocupación de aquellas 
zonas más afectadas y propiciará un reordenamiento territorial a partir de la generación de zonas de amortiguación urbana forestal en quebrada La Fábrica, además de zonas productivas, equipamiento, habitacionales, entre otras. Sus resultados aún no han sido evaluados.

EI PQMB Puertas Negras ha sido un impulsor de iniciativas bajo la mirada de la gestión y reducción del riesgo de desastre (GRD \& RRD), considerando en la preparación de su plan maestro, un estudio de micro-riegos en el sector del asentamiento informal de Pueblo Hundido con el fin de poder determinar los riesgos asociados a remociones en masa e inundaciones condicionados por su ubicación en el ápice de la quebrada La Fabrica. Pueblo Hundido se mantiene en permanente riesgo ante un evento hidrometereológico de importancia, sumado a la degradación de la parte superior por la instalación por la ocupación informal de una fábrica de ladrillos. Este estudio esta sirviendo actualmente para precisar el área de inundación determinada en el Plan Regulador Metropolitano de Valparaíso (PREMVAL), y las obras de mitigación necesarias para la radicación en condiciones seguras de las más de 70 familias que habitan el lugar hace 40 años.

Si bien, todas estas iniciativas antes mencionadas requieren de un esfuerzo de coordinación estatal, privada y comunitaria, además deben tomar un rol fundamental para la planificación estatal, tanto a nivel urbano como presupuestario en los distintos ministerios involucrados. La gestión del riesgo de desastre debe ser el eje articulador de las diferentes iniciativas de inversión estatal en territorios con altas complejidades como el sector de Puertas Negras, para que la prevención cobre la relevancia necesaria que exige un lugar como este.

\section{PUERTAS NEGRAS, DERECHO A LA VIVIENDA Y ENFOQUE DE DERECHOS EN UN SECTOR DE ALTA VULNERABILIDAD}

Bajo un enfoque de derechos, el 'derecho a la vivienda' (Cunill Grau, 2010) debe ser considerado como una obligación de los países y no como un tema que debe ser tratado bajo parámetros asistencialistas. Según lo plantea la CEPAL: "La aplicación de un enfoque de Derechos Humanos a dimensiones especiales de la protección social las ubica en el marco de los derechos exigibles, cuyos beneficiarios deben ser vistos como ciudadanos que exigen sus legítimos derechos al reclamar la asignación de recursos y la disponibilidad de servicios" (CEPAL, 2006, p.18).

En este sentido, el derecho a la vivienda puede ser reconocido como parte de los Derechos Humanos, que deben ser respetados, y a su vez, exigidos por la ciudadanía. Toda persona, sin distinción alguna, tiene derecho a vivir en un lugar que proporcione dignidad, salud física y emocional (Observatorio de Derechos Económicos Sociales y Culturales, s.f.). Este derecho se encuentra expresado en la Declaración Universal de Derechos Humanos en su artículo 17.1 "El derecho a la propiedad, individual y colectivamente" (Torres, 2017). Por su parte, el Pacto Internacional de Derechos Económicos, Sociales y Culturales (PI-DESC), también hace referencia a la vivienda, al destacar que toda persona tiene derecho a un nivel de vida adecuado, tanto en aspectos de vivienda, como de alimentación, e incluso, de vestimenta (Observatorio de Derechos Económicos Sociales y Culturales, s.f.).

La Declaración Universal de Derechos Humanos y el PIDESC se encuentran actualmente vigentes en Chile, "siendo normas aplicables mediante la habilitación constitucional del inciso $2^{\circ}$ del artículo $5^{\circ}$ de nuestra Constitución Política" (Torres, 2017). Es por esto que nos parece fundamental destacar el rol que debe tener la agenda política en relación al cumplimiento de estas normas, que por el momento se encuentran invisibilizadas $y$, muchas veces, cubiertas por estrategias asistencialistas, que no atacan el problema de raíz con un enfoque de derechos.

El caso de la Región de Valparaíso posee un escenario crítico en materia habitacional, ya que según los registros de TECHO, esta región es, desde el año 2011, la que posee mayores índices de asentamientos informales en el país, con miles de familias viviendo en terrenos tomados y con dificultad o nulo acceso a los servicios básicos (TECHO, 2016). Además, la ciudad de Valparaíso es la segunda con más campamentos dentro de la región, luego de su vecina localidad, Viña del Mar. Esto evidencia un avance insuficiente en materia habitacional en las últimas décadas por parte de las administraciones nacionales y locales. Esto se ve expresado de forma clara en el sector de Puertas Negras que posee a más de un centenar de familias viviendo en asentamientos informales (o 'campamentos' como se les conoce en Chile) que no cuentan con servicios básicos y tienen precarias redes de conectividad urbana, factor que potencia el riesgo ante una catástrofe en la zona.

Para la Fundación TECHO, en Chile, un campamento se considera cuando los asentamientos superan las ocho familias, en donde se encuentran viviendas contiguas, que se mantienen en una 
situación irregular de tenencia del terreno, por lo que carecen de acceso regular a uno o más servicios básicos de la vivienda, como son el agua potable, la electricidad y el alcantarillado (TECHO, 2016).

Como observó PQMB Puertas Negras, se puede considerar que las condiciones de las familias que viven en los asentamientos de los Lancheros, los Fleteros y Pueblo Hundido están muy alejadas de la dignidad que se exige como Derecho Humano. Se debe mencionar también que al referirse a una 'vivienda digna', no sólo se hace alusión a un lugar físico, sino, por sobre todo a un 'hábitat residencial', es decir, a un "proceso social de inclusión en el que deben integrarse el alojamiento, la privacidad, el espacio suficiente, la accesibilidad física, la seguridad en el entorno y la seguridad de tenencia" (Lentini, 2014). Por su parte, según el Comité DESC de las Naciones Unidas, una vivienda adecuada se considera cuando existe seguridad jurídica de la tenencia, disponibilidad de servicios materiales y de infraestructura, cuenta con gastos soportables y considera también, habitabilidad, accesibilidad, lugar y adecuación cultural (Observatorio de Derechos Económicos Sociales y Culturales, s.f.). Como vemos, la vivienda es en sí un bien material, y a su vez uno social que posee alto contenido simbólico (Lentini, 2014). No sólo proporciona a las personas una estructura física en donde habitar, sino por sobre todo una vida y contexto cultural que va configurando todo su contexto social. Es en este marco que avanzar en el desarrollo habitacional del polígono involucra también mejorar la conectividad urbana del lugar y trabajar en la gestión del riesgo de desastre a la hora de planificar el ordenamiento de este sector.

Desde la experiencia de Puertas Negras, garantizar la vivienda y sus servicios básicos corresponde a umbrales mínimos de bienestar, que se consideran elementales para una sociedad que pretende avanzar en términos de igualdad. En Puertas Negras, el déficit habitacional cuantitativo y cualitativo está evidenciado en la gran cantidad de personas que habitan informalmente en los asentamientos de los Lancheros y Pueblo Hundido. Para la CEPAL, "La igualdad de derechos impone límites a la desigualdad en el acceso, sobre todo cuando esa desigualdad, en cierto punto, se traduce en que parte de la sociedad se vea privada del acceso definido normativamente a partir del enfoque de la titularidad de derechos indirectamente" (CEPAL, 2010).

La vivienda, bajo el enfoque de derechos, debe proporcionar bienestar a la ciudadanía, por ende, en ningún caso debe resultar ser un perpetuador de pobreza y vulnerabilidad como se han mantenido hasta hoy en los asentamientos del país. Es fundamental que las políticas públicas y sociales en materia habitacional apunten, como lo señala la CEPAL, a "no sólo a cubrir y brindar los servicios básicos, sino también a potenciar el poder adquisitivo de los sectores más desfavorecidos para que puedan acceder a terrenos mejor ubicados, sin que ello se traduzca en un alza desmesurada de los precios de la tierra, a causa de prácticas especulativas o monopólicas" (CEPAL, 2010, p.157).

El caso de Puertas Negras permite comprender la necesidad creciente por trabajar en torno a políticas de vivienda integrales que no sólo doten de una buena infraestructura para que habiten las familias, también que estas se encuentren integradas a un entramado urbano que cumpla con las condiciones mínimas en torno a la gestión del riesgo de desastre y, de esta forma, se garantice un habitar seguro en el territorio.

Es posible crear estrategias de políticas públicas y sociales que entreguen bienestar a la ciudadanía y luchen con buenas herramientas contra la pobreza. Si bien conseguirlas implica un enorme desafío sociopolítico, estas poseen viabilidad cuando el enfoque comienza a estar centrado en los derechos. El camino hacia la transformación de políticas de vivienda, cualquiera sea la estrategia, deben estar enfocadas en su accesibilidad, asegurando su tenencia, la aptitud del terreno, la adecuación cultural, el espacio suficiente, la accesibilidad física, la privacidad y la seguridad del entorno (Lentini, 2014).

Tras el incendio de enero del 2017, las diferentes soluciones habitacionales entregadas no sólo garantizaron una construcción que cumple con las normativas, también se desarrolladó un trabajo coordinado por parte de los equipos municipales y ministeriales para propiciar un ordenamiento del territorio que permita avanzar en la prevención de riesgos, principalmente de incendios y remoción en masa.

El Programa Quiero Mi Barrio que aún se implementa en el sector ha puesto su foco en el trabajo comunitario en torno a la gestión del riesgo de desastre, intentando dar un realce a esta temática no sólo gubernamental, sino también a nivel social, con el fin de generar buenas prácticas y acciones administrativas de la mano de vecinos y vecinas del sector. 


\section{PARTICIPACIÓN COMUNITARIA COMO BASE DE LA ESTRATEGIA DE INTERVENCIÓN EN MATERIA DE GESTIÓN DEL RIESGO DE DESASTRE}

Según el departamento de Sistema de Información Geográfica (SIG) de la Municipalidad de Valparaíso, el polígono de intervención de Puertas Negras incluye las juntas vecinales N¹75 y №85, con un total de 768 hogares, de los cuales $518^{1}$ se encuentran dentro del $40 \%$ más vulnerable según del Registro Social de Hogares (RSH)2. Este registro es de gran importancia por sus valores cualitativos, ya que nos muestra el contexto social del barrio, caracterizado por tener a la mayoría de los vecinos bajo la línea de la pobreza multi-dimensional.

Tras el incendio del 2017 se mantienen las estadísticas en cuanto a la vulnerabilidad socioeconómica del sector, mientras que el Estado profundiza su inversión en el área con la llegada del Programa Quiero mi Barrio (PQMB) y la reconstrucción de viviendas (PQMB, 2018b). Actualmente, el PQMB cuenta con un monto que supera los $\$ 700$ millones de pesos chilenos (US\$ 1.022.824 del 2019), los cuales deben ser destinados mejorar los espacios comunitarios y urbanos. Los vecinos del sector, a través de la metodología participativa que propone la implementación de este programa, han priorizado diversas obras, todas directamente relacionadas a la reducción del riesgo de desastre ya que son accesos que mejoran la conectividad y posibilitan el ingreso de carros de emergencias, así también como vías de evacuación.

El contexto del lugar también jugó un rol importante para definir la estrategia de intervención del PQMB, la necesidad de estudios, y los acuerdos entre actores involucrados. El polígono en el cual se focaliza la intervención del PQMB contempla sectores consolidados (formales) y asentamientos informales o irregulares de larga data en el área, y con una extensión considerable de población. Pueblo Hundido es el segundo asentamiento más antiguo de la comuna (PQMB, 2018a). El incendio del 2017 afectó en su mayoría a familias del asentamiento que habitan en el sector de Los Lancheros del Bongo, lugar que colinda con la Quebrada La Fábrica con gran cantidad de vegetación seca en la temporada de verano.

Con este contexto de situaciones de riesgo en la zona, se acuerda la integración con el Centro de Investigación de Vulnerabilidad e Informalidades Territoriales (CINVIT) de la Escuela de Arquitectura de la Universidad de Valparaíso, con el fin de desarrollar diferentes intervenciones a nivel local y que en cuyas bases se encuentre, por un lado, la gestión del riesgo de desastre (GRD), y por otro, participación directa de vecinos y vecinas del sector. Desde entonces, el CINVIT ha desarrollado los talleres llamados DRONMAP PARTICIPATIVO, en los cuales se realizan mapeos colectivos sobre la base de información geográfica y urbana obtenida mediante drones profesionales ${ }^{3}$. EI principal objetivo de esto es generar procesos vinculantes de planificación, construcción y/o gestión entre una comunidad local y los organismos públicos y/o privados. Además, el trabajo del CINVIT entrega una visión atractiva y efectiva de cómo poder abordar la gestión del riesgo de desastre en territorios formales y/o informales como el presente en Puertas Negras.

El cumplimiento de los objetivos trazados por esta forma de trabajar la gestión del riesgo de desastre va de la mano con una comunidad organizada y consciente de cuáles son sus riesgos, además es necesario tener el compromiso vecinal por ocuparse en superar estas dificultades y generar un trabajo directo con la institucionalidad. Durante esta etapa de ejecución del programa, la comunidad presento claridad en torno a los factores de riesgo del sector y fue dotada de herramientas por parte de los equipos de trabajo en cuanto a la planificación del territorio.

El trabajo del PQMB Puertas Negras no ha estado ajeno a complejidades tanto territoriales como comunitarias, dado que durante el post-incendio se generó una fragmentación del tejido social, producto de la migración de los habitantes del territorio y las distintas soluciones habitacionales a las que cada vecino se acogió (PQMB, 2018b). Por ello, el trabajo social-comunitario se enfocó inicialmente en generar asociatividad, recuperar la participación y acción colectiva en torno a la gestión comunitaria del riesgo de incendios en el sector.

Los desafíos que deja la implementación del programa y el modelo de trabajo en el sector hacen referencia al tiempo de intervención que se plantea en el territorio. La segunda fase del programa (Fase II) es de 30 meses, aunque no responde a la realidad de los tiempos que involucra afianzar un trabajo comunitario, según quienes trabajan allí. Además, la naturaleza del funcionamiento institucional dentro del aparato estatal tiende a genera un retraso en la ejecución de las intervenciones, provocando una brecha entre la confianza establecida por la comunidad y los tiempos de materialización de las propuestas sociabilizadas. Aquí también cobra suma importancia la coordinación a nivel gubernamental y municipal que permita ejecutar un plan en el barrio de
1.Cifras entregadas por el departamento SIG, Municipalidad de Valparaíso el 2018

2. RSH, Instrumento que mide la realidad socioeconómica de las Familias.

3. http://www.cinvit.cl/dronmap 
forma oportuna, ya que una amenaza al trabajo que se levanta en Puertas Negras es el nivel de atención y prioridad que las instituciones den al sector.

\section{REFLEXIONES FINALES}

El trabajo de los equipos estatales que se han posicionado en Puertas Negras tiene gran relevancia para la comunidad, debido principalmente a los planes en torno a la mejora social y urbana del polígono de intervención. Esta labor se encuentra encabezada por el equipo de profesionales que el municipio mantiene asociados a la ejecución del Programa Quiero Mi Barrio.

El posicionamiento de la gestión del riesgo de desastre como el pilar de la intervención en el sector ha permitido generar vínculos entre las instituciones gubernamentales y la academia local para trabajar esta temática de una forma distinta a como se venía haciendo antes del 2017, y con ello resaltando el rol de los habitantes del sector. Dicho trabajo comunitario no sólo ha permitido concientizar en torno a esta materia a los vecinos y vecinas, además ha colaborado en la recomposición del tejido social que se había visto dañado tras el incendio del 2017.

El ejercicio del Derecho a la Vivienda desde un enfoque de derechos se ve afectado por el entramado urbano del sector, sumado al histórico abandono estatal de polígono. Es importante resaltar que en materia habitacional el territorio presenta grandes problemáticas, tanto en los sectores consolidados y formales, como en los asentamientos irregulares, ya que no existe un ordenamiento territorial ni intervenciones sistémicas que den soporte a soluciones sustentables y que cumplan con estregar viviendas dignas, en sus múltiples variables, considerando también a las condiciones de seguridad que necesitan las familias.

Pese a las dificultades históricas, la generación de planes estatales que trabajen la gestión del riesgo de desastre de forma planificada y coordinada entre la comunidad ha dado importantes avances en este sector, permitiendo generar acciones en torno a la prevención de incendios y potenciando la generación de estudios para el desarrollo de obras que mitiguen el riesgo. Así mismo, el Municipio de Valparaíso (2018), con la creación del Plan Maestro para la Gestión del Riesgo de Incendios, ha dado una importante señal en torno a la relevancia que debe tener para las instituciones trabajar en torno a la gestión del riesgo de desastre (GRD).

Todos los avances que se han podido desarrollar en el territorio en torno a la GRD y reducción del riesgo de desastre (RRD), guarda relación con el interés del sector público de poner en relevancia la comprensión de la urgencia de incorporar dentro del desarrollo de los territorios una política y planificación que involucre no solo la mirada de los expertos, sino también que logre recoger principalmente la mirada de los propios habitantes que han ido definiendo y surcando su habitat bajo el conocimiento intuitivo del territorio, el cual contiene el saber de cada tragedia en sus historias de vida. El rescate de este saber puede impulsar una visión conjunta que podría entregarnos lineamientos más claros y efectivos para el desarrollo de una política institucional frente al riesgo.

\section{REFERENCIAS}

Álvarez, A. (2007). Participación ciudadana y la reducción de desastres: las comunidades hacia los nuevos desafíos del desarrollo local. Revista №14. Managua: Estrategia Internacional para la Reducción de Desastres EIRD. Recuperado de: https://www.eird.org/esp/revista/no-14-2007/art33. html [visitado el 30 de Jun. 2019]

Bellec, J., \& Draghi, C. (2017). Une Nouvelle Quebrada en Fabrica'tion. Breast: Institut Géo Architecture.

Cunill Grau, N. (2010). Las políticas con enfoque de derechos y su incidencia en la institucionalidad pública. Revista del CLAD Reforma y Democracia, 46, 41-72.

CEPAL. (2006). La protección social de cara al futuro: acceso, financiamiento y solidaridad. Santiago de Chile: CEPAL.

CEPAL. (2010). La hora de la igualdad: Brechas por cerrar, caminos por abrir. Santiago de Chile: CEPAL.

Cheresky, I. (2008) Las capacidades del estado y las demandas ciudadanas: condiciones políticas para la igualdad de derechos. Buenos Aires: Programa Naciones Unidas para el Desarrollo PNUD.

García Delgado, D. (2014). Nuevos escenarios locales. El cambio en el modelo de gestión. En J. Venesia, (Ed.), Políticas públicas y desarrollo local, 1era Ed. Buenos Aires: Universidad Nacional de Quilmes, pp.10-26. 
Jirón, P. (2009). Movilidad cotidiana urbana y exclusión social urbana en Santiago de Chile. Instituto de la Vivienda, Facultad de Arquitectura y Urbanismo, Universidad de Chile [web]. Recuperado de: https://vivienda.uchilefau.cl/2013/o1/movilidad-cotidiana-urbana-y-exclusion-social-urbana-ensantiago-de-chile/ [visitado el 30 de Jun. 2019]

Lentini, M. (2012). La política de vivienda como política social. En Barreto M. \& Lentini M. (Ed.), Hacia una política integral de hábitat. Aportes para un observatorio de política habitacional en Argentina. Buenos Aires: Café de las Ciudades, pp.115-142.

Municipalidad de Valparaíso. (2018). Plan Maestro para la gestión del riesgo de incendios en la comuna de Valparaíso. Valparaíso: SECPLA, Municipalidad de Valparaíso. Recuperado de: https://www. municipalidaddevalparaiso.cl/archivos/2019/PM/Memoria_PLAN_MAESTRO_INCENDIO_2018.pdf [visitado el 30 de Jun. 2019]

United Nations International Strategy for Disaster Reduction UNISDR. (2015). Sendai Framework for Disaster Risk Reduction 2015-2030. Sendai: UNISDR.

Observatorio de Derechos Económicos Sociales y Culturales. (s.f.). Observatori DESC. Recuperado de http://observatoridesc.org/es/derecho-una-vivienda-adecuada [visitado el 30 de Jun. 2019]

Programa Quiero Mi Barrio Puertas Negras (2018a) Estudio Técnico de Bases Puertas Negras. Ilustre Municipalidad de Valparaíso y Ministerio de Vivienda y Urbanismo. Valparaíso: MINVU.

Programa Quiero Mi Barrio Puertas Negras (2018b) Informe avance segunda fase Puertas Negras. Ilustre Municipalidad de Valparaíso y Ministerio de Vivienda y Urbanismo. Valparaíso: MINVU.

Programa Quiero Mi Barrio Puertas Negras (2018c) Informe final fase I Puertas Negras. Ilustre Municipalidad de Valparaíso y Ministerio de Vivienda y Urbanismo. Valparaíso: MINVU.

Programa Quiero Mi Barrio PQMB (2019) Programa Quiero Mi Barrio, MINVU [web]. Recuperado de: http://quieromibarrio.cl/index.php/programa/ [visitado el zo de Jun. 2019]

TECHO. (2016). Chile - Catastro de Campamentos 2016. Santiago: TECHO-Chile. Recuperado de: http://datos.techo.org/dataset/catastro-campamentos-2016 [visitado el 30 de Jun. 2019]

Torres, F. (2017, 2 de Feb.). El derecho a una vivienda adecuada. El Mostrador [web]. Recuperado de: http://www.elmostrador.cl/noticias/opinion/2017/o2/o2/el-derecho-a-una-vivienda-adecuada/ [visitado el 30 de Jun. 2019]

Urbina Montaña, M. L. (1997). Algunas Consideraciones sobre un estudio de caso: Puertas Negras 1965-1980. Última Década - CIDPA, 7, 1-10. Recuperado de: http://www.cidpa.cl/wp-content/ uploads/2013/05/7.4-Urbina.pdf [visitado el 30 de Jun. 2019] 